\title{
Novel method of Automatic Recognition for Analog Measuring Instruments
}

\author{
Liu Jinqing ${ }^{1, ~ a ~}{ }^{*}$, Liu Yin ${ }^{2, b}$ and Yu Lichun ${ }^{1, c}$ \\ ${ }^{1}$ Department of information, Fuzhou College of Foreign Studies and Trade, \\ Fuzhou,Fujian, China, 350202 \\ ${ }^{2}$ College of Photonic and Electronic Engineering, Fujian Normal University, \\ Fuzhou,Fujian, China,350007 \\ aemail:jqliu82088@sohu.com, bemail:liuyin941120@ sohu.com, cemail:84246706@qq.com
}

\begin{abstract}
Keywords: Region Growing; Fuzzy Clustering; Analog Measuring Instruments; Hit-Miss Transform Abstract. Present a novel method of automatic recognition for analog measuring instruments with region growing. The whole algorithm consists of pointer extraction algorithms based on region growing method and pointer recognition algorithms based on hit-miss transform method or least square method. Besides, the seeds needed by region growing can automatically obtained by fuzzy clustering based on difference image. Experiments show that, the pointer extraction algorithms based on region growing method effectively extract the features of pointers. And the extraction algorithm lays the foundation for the Hit-Miss transform method and the least square method to obtain good recognition accuracy. The algorithm is fast and efficient, can satisfy the requirements of real-time applications. This paper first applies region growing method of image segmentation to the field of analog measuring instruments recognition, enriches the method of analog measuring instruments recognition, achieved good recognition effect.
\end{abstract}

\section{Introduction}

Analog Measuring Instruments are widely used in metallurgy, chemical industry, electric power industries because of its simple structure, easy maintenance, low cost, anti dust and water, and not affected by electromagnetic interference[1]. Especially in the wet, hot, strong radiation field, digital instruments are easy to be damaged or disturbed, which often can only use the analog measuring instruments. As those places cannot be stay for a long time for staffs, we need remote monitoring for the devices data. However, there are not RS232, GPIB or other digital interface for data transmission. Therefore, this paper presents a machine vision solution through the video image acquisition and recognition.

The first key algorithms of analog measuring instrument recognition method is segmentation algorithm for analog measuring instrument image. At present, the common segmentation algorithms for analog measuring instrument image are mainly based on difference image [2-3], based on thresholding method [4-5], based on edge extraction method [6], and based on color extraction method [7-8].

Algorithms mentioned above always only fix for a particular type of analog measuring instruments.

As a classical image segmentation algorithm of image segmentation, the image segmentation algorithm based on region has a wide range of applications the same to other segmentation algorithm. However, when we focus on the field of analog measuring instrument identification, it is almost neglected. This paper found that, in fact, the image segmentation method based on region growing has some talents which are not available in other segmentation algorithms:

First of all, the image segmentation algorithm based on region is more consistent with human 
visual characteristics. So the segmented region will have the same characteristics; Secondly, it can ensure the consistency and uniqueness of the segmentation results. Because a seed point can only achieve a region segmentation, and there are not broken fragment or blocks, which then ensures the uniqueness of the recognition results; Thirdly, it is especially suitable for the segmentation of small targets, such as the pointer in the analog measuring instrument. For the smaller the target is, the faster the segmentation will be done. Finally, the region growing algorithm is simple.

We have found an unique and important feature of analog measuring instruments : no matter how the pointer deflect $\mathrm{s}$, one point is fixed. That is the center of the pointer axis.

The unique feature of the analog measuring instruments that it got a fixed point can match well with the image segmentation algorithm based on region growing. We can just select the center of the pointer axis as the growing seed that the region growing algorithm needs, which is a good mixture of two sides. Just like a good gun with a good bullet, they can right on target together.

The number of pointers in a analog measuring instrument decides the number of the seeds need to grow. In this paper, the complex multi-pointer recognition can be realized by configuring the seed points. For a strange instrument recognition, we just need to change the position of the seed point, which have the ability to customize, and enhance the universality of the algorithm. The whole algorithm of system is mainly composed of the pointer extraction algorithms and the pointer recognition algorithms.

\section{The pointer extraction algorithms}

Figure 1. shows a steam flow meter, which has two types of pointers — 1 big black pointer and 5 small red pointers.

Plainly, the traditional methods based on difference image, threshold, edge, color or morphological method are hard to completely extract all the pointer. But the region growing method is able to crush they one by one.

Figure 2. shows the flow chart of the pointer extraction algorithms. It mainly consists of the following steps: selecting seed points, region growing, extracting ROI and filtering, image thinning and so on.

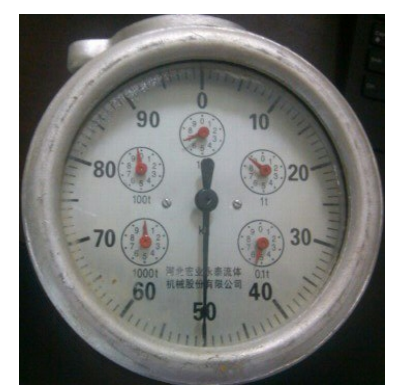

Fig.1. The analog measuring instrument used in this paper

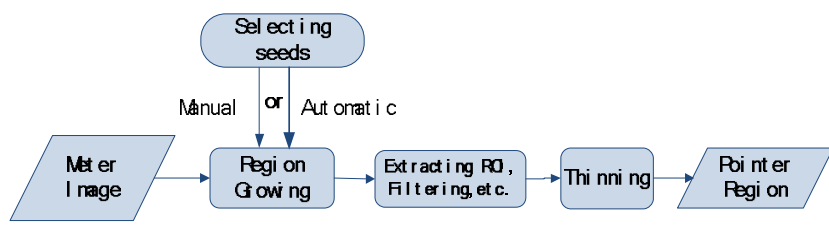

Fig.2. Flow chart of the pointer extraction algorithms

Region growing algorithm. Region growing algorithm is one of the oldest image segmentation method, which was first proposed by Levine et al. The basic idea of region growing is collect ing the pixels with similar properties together to form the final region. It can be described as the following : start with an original pixel (seed), it gradually add new similar neighboring pixels into region (just like growing) according to certain criterion until certain conditions are met, then the region growth is done. Figure 3. illustrates the process of region growing. The effect of region growing depends on three factors: (1) the selection of seeds; (2) the criterion of growth; (3) the condition of termination. 


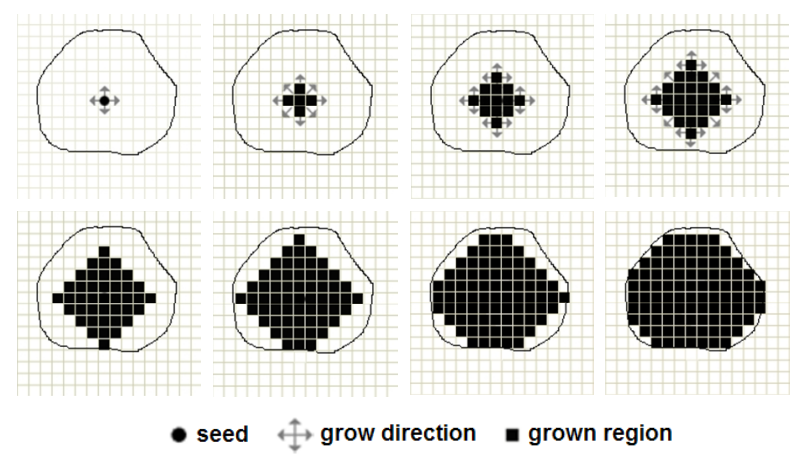

Fig.3. Sketch map of region growing process

If $I$ represent the entire image, suppose $R$ to be the grown target region, and pixel $p$ and pixel $q$ belong to image $I$. Further more, pixel $p \in R$, pixel $q \in N p, N p$ represent the neighborhood of pixel $p$ : $N p=\{q: d(p, q) \leq \rho, q \in I\}$, where $d(p, q)$ represent the distance of pixel $p$ and pixel $q, \rho$ represent Euclidean distance, usually we set $\rho=1$, namely four-connected neighborhood . It can be expected that there will be the following two categories: $\Omega p=\{q \in N p:|I(p)-I(q)| \leq T\}$ and $\bar{\Omega} p=\{q \in N p$ : $|I(p)-I(q)|>T\}$, Here, value $T$ is the threshold for judging the similarity of neighborhood, and $N p=$ $\Omega p+\bar{\Omega} p[9]$.

The traditional region growing algorithm traverses the whole image and then extract the region with the seed point as the segmentation result, which will lead to overuse of time and space. In this paper, the region growing algorithm uses Breadth-First-Search algorithm (BFS) as the pattern of growth, uses Queue's FIFO as the management of the seed points and region. After improving the growth rule and the termination condition, the region growing algorithm becomes fast, efficient and simple, which provides the possibility for the real-time applications and embedded platform.

Figure 4. shows the flow chart of region growing algorithm based on BFS. And Figure 5. shows the relative grown result.

Selection of seed points: manual and automatic. In the case of analog measuring instruments with only a single pointer, we can just set the center of the pointer axis, $\left(x_{0}, y_{0}\right)$, onto the center of whole image when installing the camera to the surface of instruments. And then select the pixel (Width/2, Height/2) as the seed point.

In the case of analog measuring instruments with multi-pointer, we can use two ways: (1) manual selection; (2) automatic selection by algorithm. This paper uses the improved algorithm based on difference image to select the seed points automatically and effectively. Figure 6. shows the selection.

How can the algorithm select the correct seed points from the difference image as Figure 6(c)? Let the foreground pixels of the difference image cluster into the expected seed points become an ideal solution. So this paper introduces the fuzzy K-means clustering algorithm into the process of selecting seed points. As a kind of clustering algorithms based on partition, fuzzy K-means clustering algorithm applies the fuzzy logic of soft computing to clustering, and it can be described as a process of optimizing the classifycation from $n$ samples of dataset $X$ to $k$ clusters, which let the sum of deviation square between clusters reach the minimum value. Namely, it's about the following mathematic problem $F$ :

$$
\min \mathrm{F}(\mathrm{W}, \mathrm{Z})=\sum_{l=1}^{k} \sum_{i=1}^{n} w_{l i}^{\alpha} d\left(Z_{l}, X_{i}\right)
$$

Where $\mathrm{W}=\left[w_{l i}\right]$ represent $k \times n$ membership matrix, $\mathrm{Z}=\left\{\mathrm{Z}_{1}, \mathrm{Z}_{2}, \ldots \mathrm{Z}_{k}\right\} \in R^{m k}$ represent the set of $k$ 
clusters, $d(\cdot, \cdot)$ values the square of the Euclidean norm。Due to limited space, further information about fuzzy clustering algorithms, 10 refs. Fuzzy clustering algorithm can improve the convergence of the clustering, so as to improve the accuracy of automatic selection of seed points.

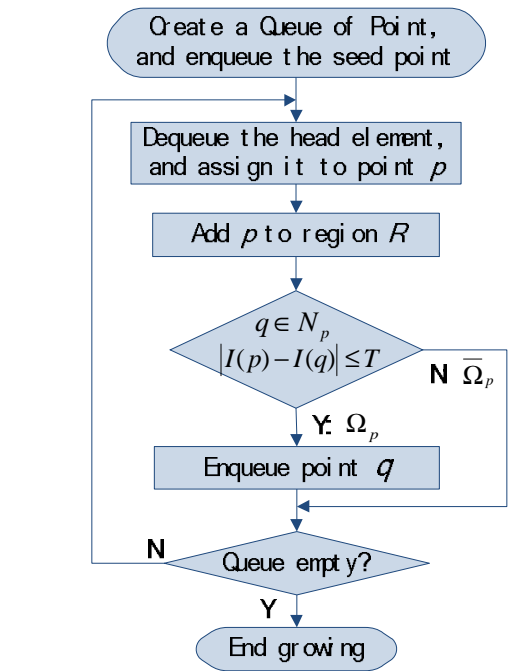

Fig.4. Flow chart of region growing algorithm based on BFS

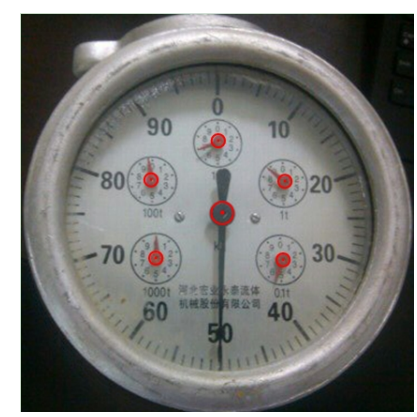

(a) The effective range of the seed points

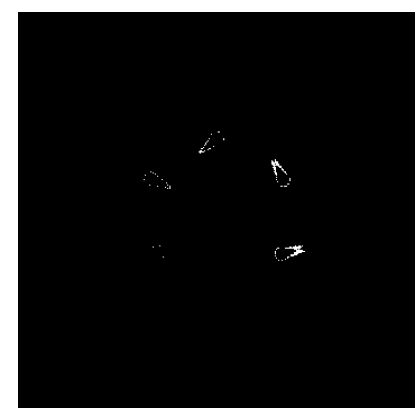

(c) automatic selection : difference image

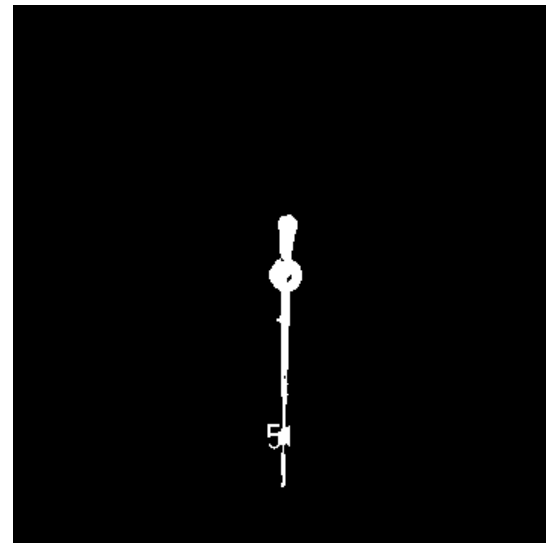

Fig.5. Segmentation result using region growing algorithm

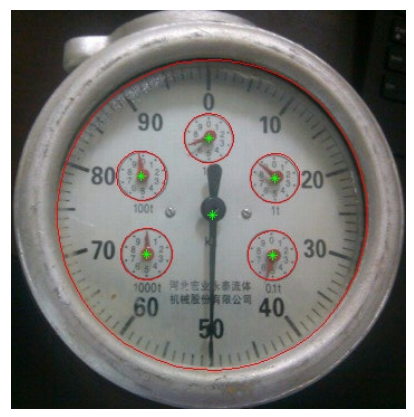

(b) manual selection

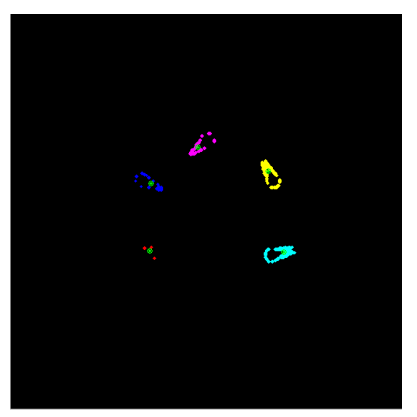

(d) automatic selection : fuzzy clustering

Fig.6. The selection of seed points

As shown in Figure 6 (d), after clustering the foreground pixels of difference image, there will be 5 clusters and each cluster has its own center or centroid. Different colors represent different clusters, and the centroids are marked with green points.

Extracting the Region of Interest. As the region growing algorithm only grow in the area of pointers, and the area of pointers account for only a very small proportion of the whole image, it's suggested to extract the area of pointers as our Region Of Interest (ROI). It can avoid the unnecessary computational cost and speed up the algorithm. Figure 7 (a) shows the extraction result of ROI.

Image thinning. Image thinning is the operation to delete the edge pixels of the image until the 
skeleton (one-pixel) left under maintaining the original shape. It helps to highlight the shape features and reduce the amount of redundant information, which could improve the performance of automatic recognition effectively.

There are kinds of image thinning algorithms. After testing, it shows that the Hilditch thinning algorithm is easy to produce burr, the Pavlidis and Rosenfeld thinning algorithm results deviate from the center of axis. In this paper, the thinning algorithm based on Look-Up Table was applied. Look-up table thinning algorithm makes a lookup table based on human subjective thinning rules. By avoiding kinds of judgment operation, the speed of its algorithm is faster than the other thinning algorithm, and it got a satisfied thinning effect.

Suppose there is a pixel $p$ in the image waiting for thinning, it needs to look up the precomputed table, then decide to delete the pixel or not. The rules of making the precomputed lookup table uses the following algorithm :

In the first subiteration, delete pixel $p$ if and only if the conditions $\mathrm{G} 1, \mathrm{G} 2$, and $\mathrm{G} 3$ are all satisfied.

In the second subiteration, delete pixel $p$ if and only if the conditions G1, G2, and G3' are all satisfied. Details show in [10] refs.

Figure 7 (b) shows the image thinning result by look-up table thinning algorithm. We can see that the linear features is obvious, which lay the foundation for the subsequent pointer recognition.

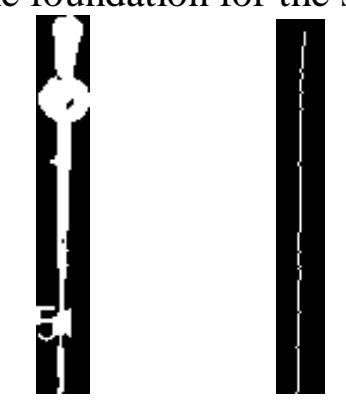

(a) Extracting the ROI

(b) Image thinning

Fig.7. Extracting the ROI and image thinning

\section{The pointer recognition algorithms}

The Aim of pointer recognition algorithms is to get the pointer reading, while the pointer reading is corresponding to the angle or line of pointer. Therefore, the key of pointer recognition lies in the line detection or angle detection.

The common line detection algorithms have the following: (1)detecting lines by Hough transform method; (2) fitting lines by least squares fitting method. Besides, this paper found that the morphological Hit-Miss transform method can be used to hit the lines. Therefore, the following will focus on these three methods.

In Hough transform method, the original image space coordinates $(x, y)$ is mapped to the parameter space $(\rho, \theta)$, so the curve detection problem in the original image is transformed into searching peak in parameter space. Hough transform makes the detection much more simple by transforming the global features detection into the local features detection [11]. Hough transform method use the following normal representation to detect a line:

$$
\rho=x \cos (\theta)+y \sin (\theta)
$$

The least squares method is a mathematical optimization method by minimizing the sum of error squares to find out the best parameter of the line. It can be used for curve fitting. When used for fitting a line, the line function is defined as $y=a+b x$, Here, the known quantity are $N$ foreground pixels $\left(x_{i}, y_{i}\right)$, 
$i=1,2, \ldots, N$, and the unknown quantity are the line parameter $a$ and $b$. The solution of $a$ and $b$ meeting the condition that the sum of error squares $\sum\left[y_{\mathrm{i}}-\left(a+b x_{\mathrm{i}}\right)\right]^{2}$ reach the minimum is: [12]

$$
\begin{gathered}
\hat{a}=\frac{\left(\sum x_{i}^{2}\right)\left(\sum y_{i}\right)-\left(\sum x_{i}\right)\left(\sum x_{i} y_{i}\right)}{N\left(\sum x_{i}^{2}\right)-\left(\sum x_{i}\right)^{2}} \\
\hat{b}=\frac{N\left(\sum x_{i} y_{i}\right)-\left(\sum x_{i}\right)\left(\sum y_{i}\right)}{N\left(\sum x_{i}^{2}\right)-\left(\sum x_{i}\right)^{2}}
\end{gathered}
$$

The morphological Hit-Miss transform is a basic tool for shape detection. It can detect different shapes by choosing different structure elements [13]. This paper use Hit-Miss transform to detect the endpoints of the thinned pointer image. Firstly, Let the thinned pointer image as Figure 7 (b) shown do Hit-Miss transform with the 8 kinds of Structure Elements as Figure 8 shown respectively. Then superimpose all the binary images after Hit-Miss transform by OR operation. In this way, we can detect all the endpoints of all directions, namely hit the two endpoints of the line. Finally, for two points determine a straight line, we can calculate the angle of the line.

$$
\begin{aligned}
& \begin{array}{|l|l|l|}
\hline 0 & 1 & 0 \\
\hline 0 & 1 & 0 \\
\hline 0 & 0 & 0 \\
\hline
\end{array} \quad \begin{array}{l|l|l|}
\hline 0 & 0 & 1 \\
\hline 0 & 1 & 0 \\
\hline 0 & 0 & 0 \\
\hline 0
\end{array} \quad \begin{array}{ll|l|l|}
\hline 0 & 0 & 0 \\
\hline 0 & 1 & 1 \\
\hline 0 & 0 & 0 \\
\hline
\end{array} \quad \begin{array}{lll|l|}
\hline 0 & 0 & 0 \\
\hline 0 & 1 & 0 \\
\hline 0 & 0 & 1 \\
\hline
\end{array} \\
& \begin{array}{|l|l|l|}
\hline 0 & 0 & 0 \\
\hline 0 & 1 & 0 \\
\hline 0 & 1 & 0 \\
\hline
\end{array} \quad \begin{array}{lll|l}
\hline 0 & 0 & 0 \\
\hline 0 & 1 & 0 \\
1 & 0 & 0 \\
\hline
\end{array} \quad \begin{array}{ll|l|l|}
\hline 0 & 0 & 0 \\
\hline 1 & 1 & 0 \\
0 & 0 & 0 \\
\hline
\end{array} \quad \begin{array}{llll|}
\hline 1 & 0 & 0 \\
\hline 0 & 1 & 0 \\
\hline 0 & 0 & 0 \\
\hline
\end{array}
\end{aligned}
$$

Fig.8. The 8 Structure Elements used in the Hit-Miss transform

\section{Recognition results \& analysis}

Figure 9. shows the recognition results of the above methods. And figure 10. shows the error analysis. It can be concluded as follow :

The Hough transform method is hard to detect all the pointers, especially for the small points. Because one pair of Hough transform parameters is hard to adapt to all situations.

The least squares method and the Hit-Miss transform method both have good recognition precision of pointer when the image quality is suitable, but the two are kind of different :

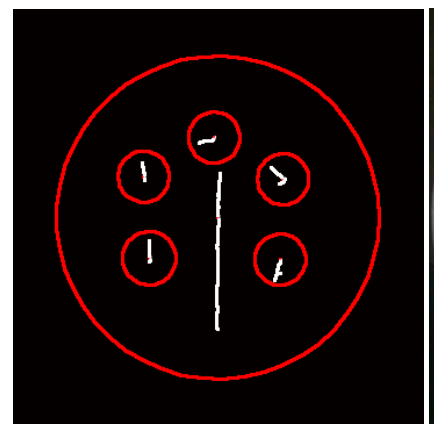

a) The effect of pointers extraction

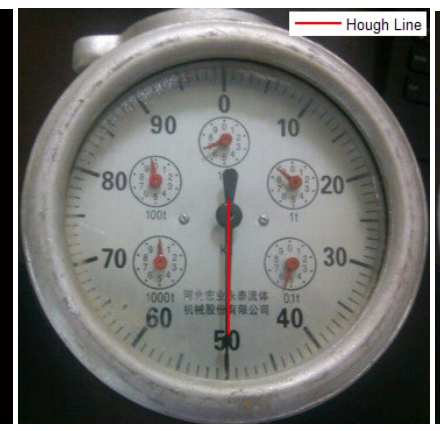

b) Hough transform method

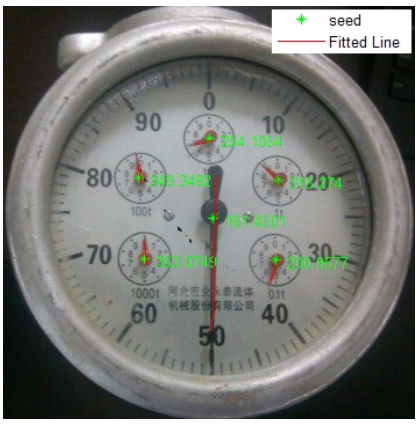

c) The least squares method

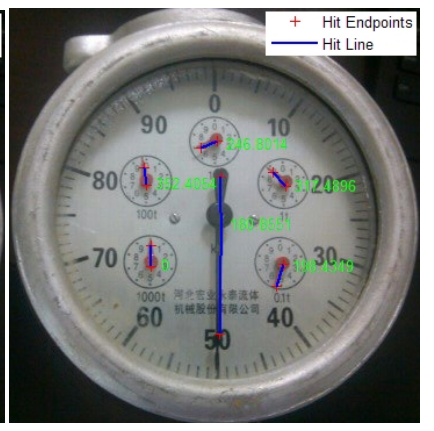

d) the Hit-Miss transform method

Fig.9. Pointer recognition results of three methods (green labels are recognized angles) 
The least squares method for fitting lines is a pure mathematical tools without any uncertain parameters. It has properties of relatively low error, relatively high precision, relatively good robustness and allowing a certain degree of noise.

The Hit-Miss transform method for hiting lines can apply to multi-pointer recogintion and ensure the uniqueness of recogintion results. When the noise or lighting is quite serious, it will cause the offset and distortion of pointer to some extent. But the extent of endpoints damaged is much less than that of the main middle part. Therefore, in this condition, the Hit-Miss transform method has more accurate recognition rate than the least squares method.

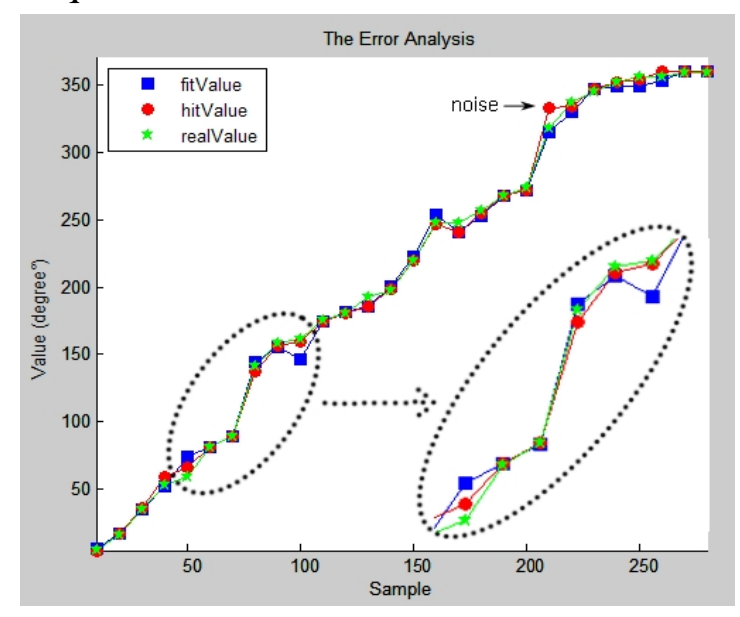

Fig.10. Error analysis of FitLine and HitLine method

Table 1. The recognition error of analog measuring instrument

\begin{tabular}{ccccc}
\hline \multirow{2}{*}{ ERR \% } & \multicolumn{3}{c}{ Pointer Types } \\
\cline { 2 - 5 } & & Small Pointers & Big Pointers & Avg \\
\hline \multirow{2}{*}{$\underset{8}{\overrightarrow{8}}$} & FitLine & 1.33 & 0.14 & 1.14 \\
\cline { 2 - 5 } & HitLine & 0.97 & 0.10 & 0.83 \\
\cline { 2 - 5 } & Avg & 1.15 & 0.12 & -
\end{tabular}

Table 1. shows the recognition error of analog measuring instrument in this paper. Where, in the case of different point types, the big black pointer performs obviously much better than the small red points; And in the case of different recognition methods, the Hit-Miss transform method (HitLine) performs slightly better than the least squares method (FitLine) in current experimental environment.

\section{Conclusion}

In this paper, the pointer extraction algorithms based on region growing gets a good effect, which is applied to the field of automatic recognition of analog measuring instruments for the first time. It enrichs the application methods of analog measuring instruments recognition. Experiments show that the algorithm can adapt to various types of analog measuring instruments. And its high efficiency can meet the real-time application. Besides, the Hit-Miss transform method and the least squares method both perform well as pointer recognition algorithms, each has its own merits.

The above algorithms have been verified on Matlab platform and then transplanted successfully to the OpenCV platform for higher speed; And they are able to run on the embedded hardware platform based on Android operating system finally for wider application. 


\section{Acknowledgment}

The authors gratefully acknowledge the supports from The National Natural Science Foundation of China(61179011), Natural Science Foundation of Fujian Province(2010J01327), Fujian Provincial Key Laboratory of Photonics Technology and Fujian Education Department Technology Foundation (JB07040).

The authors also acknowledge the editor and colleagues who provided technical supports.

\section{References}

[1] Song R J, Zhao L Y, Improved Hough Transform and Its Application in Automatic Recognition on Dial Instrument in Power Plants [J], Journal of Northeast Dianli University, 2011,31(3):42-46.

[2] F.Correa Alegria, A.Cruzz.Serra, Automatic Calibration of Analog and Digital Measuring Instruments Using Computer Vision, IEEE Transaction on Instrumentation and Measurement, 2000, 49(1):94-99.

[3] Jin J Q, Liu Q, Research of Recognition for Pointer Angle of Dial Based on Embedded ARM [J], Journal of Nanjing Normal University(Engineering and technology edition), 2009, 9(1):23-27.

[4] Xue J R, Yang M, A Method of Automatic Indication Recognition for A Point Meter by Image Processing [J], China Instrumentation , 2005, 7(10) : 73-82.

[5] REN G L, Fast segmentation algorithm for image of gauge with pointer [J], Computer Engineering and Design, 2005, 26(3):790-792.

[6] Chen J L, You L H, Zhang Q J, The Applization of Digital Image Drocessing Technology in the Reading System for Analog Meter [J], Journal of Southern Yangtze University , 2005, 4(6) : 611-614.

[7] Sun F J, An T J, Fan J Q, et al, Study on the Recognition of Pointer Position of Electric Power Transformer Temperature Meter [J], Proceedings of the CSEE , 2007, 27(7) : 70-75.

[8] Ji C Y, Kan Y F, Jiao S X, Recognition of Instrument Color Pointer Based on Color Extracting Method [J]. Journal of Northeast Dianli University , 2012 , 32(3) : 79-82.

[9] Jundi D, Runing M, Songcan C, A scale-based coherence connected tree algorithm for image segmentation, IEEE Transactions on Image Processing, 2008,17(2):204-216.

[10]Louisa L, Lee SW, Suen CY, Thinning methodologies-a comprehensive survey Pattern Analysis and Machine Intelligence , IEEE Transactions, 1992, 14(9):869-885.

[11] Matas J, Galambos C, Kittler J, Robust detection of lines using progressive probabilistic Hough transform, Computer Vision and Image Understanding, 2000, 78(1):119-137.

[12] Yi D Y, Shen Y B , Li Y F, Computational Method [M], 2nd.ZheJiang: ZheJiang University Press , $2002: 71-82$.

[13] Gonzalez R C, Woods R E, Digital Image Processing [M], 3nd.BeiJing: Publishing House of Electronics Industry, 2011:411-412. 
